\title{
Graphene on the pilot line
}

\author{
The next stage of the Graphene Flagship is marked by a $€ 20$ million investment for an experimental Pilot Line, \\ promoting the transition of devices based on two-dimensional materials from research to industry.
}

$t$ is rare for newly discovered materials to hit the market in just a few years. Yet, the 2010 Nobel Prize in Physics and the launch of the Graphene Flagship (GF) project $^{1}$ by the European Commission in 2013 have set the technological expectations for graphene very high. The main goal of this initiative, which was awarded an impressive budget of $€ 1$ billion, is to support an academic-industrial consortium of partners in order to take graphene from academic laboratories into society in ten years $^{1}$. Similar worldwide initiatives have funded the early innovation work necessary to climb the technology readiness level (TRL) ladder, a list of nine indicators that describe the degree of maturity of a new technology - the higher the TRL, the closer a technology is to commercialization. In 2015, South Korea announced a five-year technology roadmap to boost the commercialization of graphene with an investment of US\$107.7 million (ref. ${ }^{2}$ ). The Chinese government issued a set of policies that identified graphene industrialization as a priority in the Development Plan for New Materials during the 13th Five-year Plan Period (2016-2020) ${ }^{3}$. In 2014, IBM announced a US $\$ 3$ billion investment towards the development of next-generation chip technology including two-dimensional (2D) materials - to complement silicon ${ }^{4}$. This global effort has been driven by the development of large-scale synthesis methods ${ }^{5,6}$, and by the lab-scale realization of several device prototypes for (opto-)electronics, chemical and biomedical sensing, flexible electronics and photovoltaics ${ }^{6}$. It was not long before an ever-growing class of TRL- 4 devices emerged, indicative of technologies validated in laboratory settings. And soon after that, high-end applications like touch screens for mobile phones, mechanically and thermally enhanced composites, automotive components or even tennis rackets ${ }^{6,7}$ saw small-to-medium-scale commercialization.

However, until today, the market demand for 2D-material-based products has been hindered by a number of issues. First, the current state of the 2D materials supply industry is still perceived as immature by potential customers, lacking sufficient standardization, and quality and performance reliability of the produced materials ${ }^{7}$. Furthermore, in order to

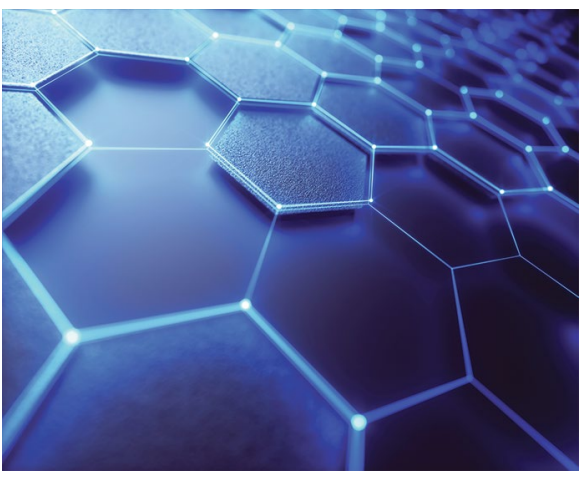

ktsdesign / Science Photo Library.

attract investors ${ }^{6,7}, 2 \mathrm{D}$ materials should either provide orders-of-magnitude enhancements in device performance relative to state-of-the-art technologies, or target entirely new market shares where the business demands cannot be fulfilled by existing technologies. A promising direction is offered by graphene optoelectronic devices with broadband high-speed operation and low power consumption ${ }^{8}$, which also have the potential to be integrated with silicon complementary metal-oxidesemiconductor (CMOS) technology $y^{5,8}$.

The European Commission is now launching a $€ 20$ million investment for the realization of the GF 2D Experimental Pilot Line $^{9}$, aiming at advancing the development of 2D electronics, optoelectronics and sensing towards industrialization. The core idea is to build a bridge between university researchers, spin-offs, and industry by transferring the existing GF know-how to an industrial environment. The initiative will work towards the integration of 2D materials into established semiconductor platforms such as silicon, creating opportunities for hybrid electronic technologies that leverage the best of both worlds. Enabling manufacturers to work at the interface between silicon and 2D materials in the Pilot Line might help overcome the challenge of integrating materials having different properties, both in terms of dimensionality and electronic functionalities.

The consortium involved in the project, consisting of European manufacturers and semiconductor fabrication lines, will build an industrially compatible infrastructure for the production and manufacturing scale-up of $2 \mathrm{D}$ devices with TRLs ranging from 7 to 8 (corresponding to a complete system prototype in its operational environment). The Pilot Line builds on the existing GF partners and infrastructure, with the long-term plan to develop microelectronic 'foundries' distributed across several industrial partners that take charge of the production steps covering the entire value chain of an integrated circuit. Some of the stakeholders involved (IMEC, VTT and AMO, to mention a few) offer the competitive advantage of being already equipped to process materials beyond CMOS - something a traditional manufacturer typically does not.

The $€ 20$ million investment from the European Commission (with 50\% of the budget allocated to personnel and the other $50 \%$ to infrastructure development) should allow the Pilot Line to sustain itself by 2024 . After that, the foundry will operate as an on-demand platform, providing services to users (academics, research centres and companies) for a fee. Device prototyping and testing will be among the services offered, so ideally any stakeholders could develop and test their technologies based on 2D materials. Ultimately, modules for the design and manufacturing of several basic building blocks for 2D technologies will be made publicly available for European users through multi-purpose wafers.

In spite of its European genesis, should this initiative be successful, its envisaged impact in R\&D and industry may foster the growth of a global industrial ecosystem for $2 \mathrm{D}$ materials.

Published online: 28 April 2021

https://doi.org/10.1038/s41563-021-00999-0

References

1. The Graphene Flagship https://go.nature.com/3dbdXrD

2. Graphene commercialization promotion technology roadmap. Republic of Korea Ministry of Culture, Sports and Tourism (8 April 2015); https://go.nature.com/3tby7HJ

3. Global and China Graphene Industry Report, 2019-2025 (Research and Markets, 2019).

4. IBM announces $\$ 3$ billion research initiative to tackle chip grand challenges for cloud and big data systems. IBM (10 July 2014); https://go.nature.com/3mDQOBk

5. 2D materials from lab to Industry. Nature Materials (29 May 2019); https://www.nature.com/collections/igjcccedji

6. Graphene 15. Nature Nanotechnology (3 October 2019); https://www.nature.com/collections/jegddcihic Döscher, H. et al. 2D Mater. 8, 022005 (2021).

8. Romagnoli, M. et al. Nat. Rev. Mater. 3, 392-414 (2018).

9. Experimental pilot line for devices based on graphene, related 2D materials and heterostructures. CORDIS (4 July 2019); https://go.nature.com/3wIMLsd 